\title{
Chemische Untersuchungen von gesunden und rhachitischen Knochen.

\author{
Von
}

Th. GaBmann, Zürich.

(Der Redaktion zugegangen am 6. November 1910.)

Vielfach und mannigfaltig sind die Forschungen nach den Ursachen einer weit verbreiteten Knochenkrankheit, der Rhachitis. Ich will die Arbeiten, die bereits hierüber publiziert worden sind, nicht erwähnen, da sie zumeist biologischer oder histologischer Art sind, somit einer Einleitung meiner rein chemischen, analytischen Arbeit nicht entsprechen würden. Was bis jetzt über das Wesen dieser Krankheit bekannt ist, gipfelt darin, daß der rhachitische Knochen ärmer an Kalk ist als der normale. Damit ist allerdings das Bild des kranken Knochens noch lange nicht erschöpft, vielmehr hoffte ich nicht nur durch Feststellung des Kalkgehaltes, sondern auch der übrigen Bestandteile sowohl des gesunden wie rhachitischen Knochens und Vergleichung der Resultate weitere Anhaltspunkte zur Beurteilung der Rhachitis herausschälen zu können.

Wegleitend in dieser Beziehung waren mir fernerhin auch einige Fragen, die mir bei Beginn dieser Arbeit vorgeschwebt haben, nämlich: Sind außer beim Kalk auch bei den übrigen Bestandteilen bedeutsame quantitative Unterschiede zu erkennen? Ist nach den vorhandenen Analysenresultaten eine Verteilung der Säuren auf die Basen möglich? Ist der gesamte rhachitische Knochen anormal aufgebaut oder trifft dies nur partiell zu?

Nicht ohne Interesse war für mich der Gedanke, wenigstens den Stickstoffgehalt in beiden Knochen festzustellen, um auch darin eventuell Beweismaterial für die Veränderung des Knochens $\mathrm{zu}$ finden. 
Um experimentell die Aufgabe durchzuführen, war die Auswahl der Knochen von Wichtigkeit. Hierbei hat mich Herr Prof. Dr. Schmidt, Direktor des pathologischen Institutes der Universität Zürich, durch Zuwendung von passenden Knochenstücken, Rippenknochen, in zweckmäßiger und erfolgreicher Weise unterstützt, dem ich an dieser Stelle meinen verbindlichsten Dank ausspreche. Die Vorbereitung der Knochen zur Analyse geschah in der Weise, daß dieselben vorerst von allen nicht zum eigentlichen Knochen gehörenden Stoffen befreit und mittels Wasser und Äther gut gereinigt wurden. Nach längerem Trocknen an der Luft wurde der Knochen alsdann fein pulverisiert und bei $100-200^{\circ}$ im Luftbad zum konstanten Gewicht gebracht.

Die Farbe der pulverisierten Knochen war merklich verschieden, die des normalen gelblich mit Stich ins Graue, die des rhachitischen weißlich mit Stich ins Graue. Nach dem Trocknen im Luftbade bei $100-120^{\circ}$ war keine Veränderung der Farbe zu konstatieren. Die nachfolgenden Daten werden nunmehr den analytischen Befund nachweisen.

\section{Normale (gesunde) Knochen. ${ }^{1}$ )}

I. Analyse.

0,2853 g Knochensubstanz gaben bei Trocknung auf $110-120^{\circ}$

$$
0,0333 \mathrm{~g} \mathrm{H}_{2} \mathrm{O}=11,67 \% \mathrm{H}_{2} \mathrm{O} \text {. }
$$

$0,1879 \mathrm{~g}$ getrockn. Knochensubst. gaben $0,0696 \mathrm{~g}$ Glühverlust $=37,04 \%$ 0,1879 , \ $, 0,0650, \mathrm{CaO}=\mathrm{Ca}=24,48 \%$ 0,1879 . $>\quad$ ॰ $0,0004 \times \mathrm{MgO}=\mathrm{Mg}=0,10 \%$ 0,1879 > \ $>0,0475 \gg \mathrm{P}_{2} \mathrm{O}_{5}=\mathrm{PO}_{4}=33,79 \%$ 0,2340 ॰ \ $, 0,0055, \mathrm{CO}_{2}=\mathrm{CO}_{3}=3,20 \%$ 0,1262 > $\gg 0,0005 \times \mathrm{Cl}=0,39 \%$ 0,1650 > \ > $\quad, 0036 \diamond \mathrm{K}_{2} \mathrm{PtCl}_{6}=\mathrm{K}=0,30 \%$ 0,1650 > $\gg \quad 0,0033 \times \mathrm{Na}_{2} \mathrm{SO}_{4}=\mathrm{Na}=\frac{0,60 \%}{99,90 \%}$

II. Analyse.

$0,1510 \mathrm{~g}$ Knochensubstanz gaben bei Trocknung auf $110-120^{\circ}$ 0,0173 g $_{8} \mathrm{O}=11,45 \% \mathrm{H}_{8} \mathrm{O}$.

1) Obige Knochen entstammen normalen Rippen eines $2^{1 / 2}$ jährigen Knaben. 
Untersuchungen von gesunden und rhachitischen Knochen. 163

$0,1308 \mathrm{~g}$ getrockn. Knochensubst. gaben $0,0487 \mathrm{~g}$ Glühverlust $=37,23 \%$

\begin{tabular}{|c|c|c|c|c|c|c|}
\hline 0,1308 ? & ? & 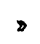 & & $0,0446>\mathrm{CaO}$ & $=\mathrm{Ca}$ & $=24,31$ \\
\hline 0,1308 , & , & D & , & $0,0003>\mathrm{MgO}$ & $=\mathrm{Mg}$ & $=0,10$ \\
\hline 0,1308 , & ه & , & , & $0,0329>\mathrm{P}_{2} \mathrm{O}_{6}$ & $=\mathrm{PO}_{4}$ & $=33,33$ \\
\hline 0,1822 , & » & D & $\triangleright$ & $0,0041 \cdot \mathrm{CO}_{8}$ & $=\mathrm{CO}_{\mathrm{s}}$ & $=3,01$ \\
\hline 0,1068 , & D & , & , & 0,0005 • $\mathrm{Cl}$ & & $=0,46$ \\
\hline 0,1760 , & , & , & $\triangleright$ & $0,0039>\mathrm{K}_{2} \mathrm{PtCl}$ & ${ }_{8}=\mathrm{K}$ & $=0,30$ \\
\hline 0,1760 . & , & , & $\triangleright$ & $0,0037 \mathrm{~g} \mathrm{Na}_{8} \mathrm{SO}$ & $4=\mathrm{Na}$ & $=0,68$ \\
\hline
\end{tabular}

Rhachitische Knochen. $\left.{ }^{1}\right)$

I. Analyse.

0,3046 g Knochensubstanz gaben bei Trocknung auf 110-120

$$
0,0324 \mathrm{~g} \mathrm{H}_{2} \mathrm{O}=10,63 \% \mathrm{H}_{2} \mathrm{O} \text {. }
$$

$0,1307 \mathrm{~g}$ getrockn. Knochensubst. gaben $0,0561 \mathrm{~g}$ Glühverlust $=42,92 \%$

\begin{tabular}{|c|c|c|c|c|c|c|}
\hline 0,1307 ， & D & > & D & $0,0391 \gg \mathrm{CaO}$ & $=\mathrm{Ca}$ & $=21,34 \%$ \\
\hline 0,1307 , & $\Rightarrow$ & , & 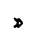 & $0,0013>\mathrm{MgO}$ & $=\mathrm{Mg}$ & $=0,53 \%$ \\
\hline 0,1307 , & $\triangleright$ & ? & , & $0,0299>\mathrm{P}_{8} \mathrm{O}_{5}$ & $=\mathrm{PO}_{4}$ & $=30,22 \%$ \\
\hline 0,1796 • & , & , & D & $0,0035 \gg \mathrm{CO}_{2}$ & $=\mathrm{CO}_{3}$ & $=2,61 \%$ \\
\hline 0,1412 ㅇ & 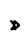 & D & > & $0,0006, \mathrm{Cl}$ & & $=0,45 \%$ \\
\hline 0,1898 , & , & D & , & $0,0042, \mathrm{~K}_{\mathbf{2}} \mathrm{PtC}$ & $6=\mathrm{K}$ & $=0,31 \%$ \\
\hline 0,1898 , & , & ه & » & $0,0046 \cdot \mathrm{Na}_{2} \mathrm{SC}$ & $4=\mathrm{Na}$ & $=0,73 \%$ \\
\hline
\end{tabular}

II. Analyse.

0,4028 g Knochensubstanz gaben bei Trocknung auf 110-120 0,0434 g $_{2} \mathrm{O}=10,77 \% \mathrm{H}_{2} \mathrm{O}$.

$0,2151 \mathrm{~g}$ getrockn. Knochensubst. gaben $0,0915 \mathrm{~g}$ Glühverlust $=42,53 \%$

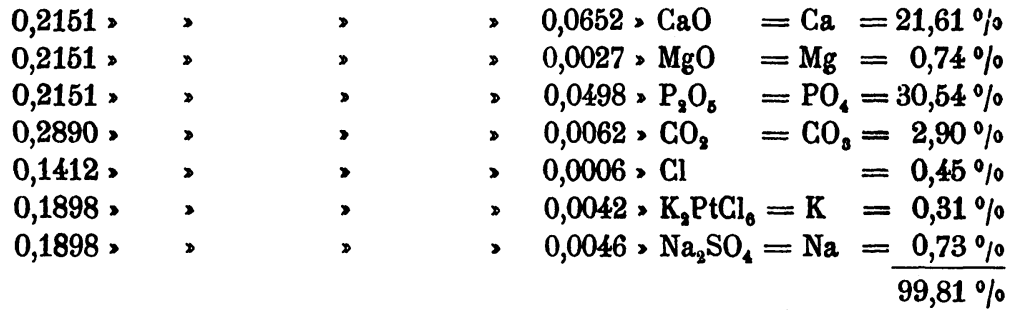

Wie aus den analytischen Daten ersichtlich ist, besteht zwischen normalen und rhachitischen Knochen in ihren Analysenresultaten eine recht auffallende Verschiedenheit. Schon beim Wassergehalt setzt dieselbe ein und berührt die Mehrzahl der

1) Obige Knochen entstammen rhachitischen Rippen eines $1^{1 / 2}$ jährigen Knaben. 
erhaltenen Ergebnisse. In meinen Zahnanalysen habe ich bereits darauf hingewiesen, daß zwischen Kalk und Wasser konstante Beziehungen herrschen. Es erübrigt mir, auch hier auf diese Erscheinung aufmerksam zu machen. Vergleicht man die Abnahme des Wassergehaltes mit derjenigen des Kalkes beim rhachitischen Knochen, so ist es recht interessant $\mathrm{zu}$ konstatieren, daß eigentlich beide annähernd in demselben Verhältnis abnehmen.

Solche prägnante Ergebnisse lassen doch die Annahme $\mathrm{zu}$, daß wir im Knochen eine Verbindung besitzen, die sogenanntes Konstitutionswasser enthält. Wir werden später bei der Besprechung der Analysen des Kalkes, der Phosphorsäure und der Kohlensäure sehen, daß die Möglichkeit von Wasser enthaltenden Verbindungen im Knochen nicht ausgeschlossen ist. Recht in die Augen springend ist der Verlust an Kalk und Phosphorsäure beim rhachitischen Knochen, etwas weniger derjenige der Kohlensäure, immerhin so, daß die Abnahme der Werte von Kalk, Phosphorsäure und Kohlensäure in einem bestimmten Verhältnis erfolgt. Bei näherer Prüfung desselben ergibt sich, daß die Werte von Kalk, Phosphorsäure und Kohlensäure in gleichem Verhältnis zueinander stehen wie beim normalen Knochen, nur mit dem Unterschiede, daß die Werte von Kalk, Phosphorsäure und Kohlensäure kleiner sind als beim normalen Knochen. Unwillkürlich ruft das aber die Ansicht hervor, $\mathrm{da} B$ im gesunden und rhachitischen Knochen die gleiche Verbindung vorhanden ist, bestehend aus den Faktoren: Kalk, Phosphorsäure und Kohlensäure. Ich werde später bei Verteilung der Säuren auf die Basen auf diese wichtige Tatsache zurückkommen. Diese Stabilität des gegenseitigen Verhältnisses von Kalk, Phosphorsäure und Kohlensäure spricht auch dafür, daß der Rhachitis als Ursache nicht eine allmähliche Zersetzung des Knochens zu eigen ist. Wäre dies der Fall, so würde obiges Verhältnis in dieser Vollendung nicht denkbar sein, vielmehr würde die Analyse da und dort Verschiebungen aufweisen, die diese Krankheit als Auflösungsprodukt charakterisieren würde.

Die in der Literatur verbreitete Tatsache, daß Kalkmangel 
die Ursache der Knochenrhachitis sei, muß nunmehr notwendigerweise dahin ergänzt werden, daß sich in gleicher Weise auch Phosphorsäure und Kohlensäure daran beteiligen; dadurch sind wir in der Lage, ein viel zutreffenderes, kompakteres Bild vom Wesen dieser Krankheit zu erhalten. Eine in pathologischer Hinsicht wichtige Beobachtung folgert aus der vermehrten Anwesenheit des Magnesiums im rhachitischen Knochen. Es ist im höchsten Grade auffallend, daß gerade der rhachitische Knochen mehr Magnesium besitzt als der normale. Eine derartige Erscheinung habe ich auch in meinen Zahnanalysen nachweisen können. Dort hat es sich ergeben, daß der Bestand an Magnesium in unseren heutigen Zähnen, ${ }^{1}$ ) die an Widerstandsfähigkeit eingebüßt haben, ganz erheblich denjenigen in prähistorischen Menschenzähnen ${ }^{2}$ ) übertrifft. Was soll diese Analogie bedeuten? Ich kann mir keine andere Erklärung $\mathrm{zu}-$ recht legen, als daß dies eine abnormale Erscheinung ist, ein Moment, das darauf hinausläuft, unsern Organismus krankhaften Einflüssen auszusetzen. Das können wir mit Sicherheit sagen, $\mathrm{da} B$ der vermehrte Magnesiumgehalt da auftritt, wo wir Abweichungen vom normalen Knochentypus voraussagen können.

In bezug auf ChIor, Kalium und Natrium ist zu bemerken, daß keine Veränderung des Gehaltes eingetreten ist.

Beim rhachitischen Knochen ist der Gehalt an organischer Substanz um 5\% vermehrt, trotzdem verfügen beide Knochen über die gleiche Menge Stickstoff, wie aus folgender Elementaranalyse nach Dumas erhellt:

Normaler Knochen: 0,1168 g Substanz gaben bei 6,2 Volumen, $17^{\circ}$ Temperatur und $717 \mathrm{~mm}$ Barometer $=5,8 \% \mathrm{~N}$.

Rhachitischer Knochen: 0,1362 g Substanz gaben bei 7,4 Volumen, $16^{\circ}$ Temperatur und $723 \mathrm{~mm}$ Barometer $=6,01 \% \mathrm{~N}$.

Verteilung der Säuren auf die Basen.

Bei der Besprechung der einzelnen Analysenresultate haben wir gesehen, daß nicht nur beim normalen, sondern

1) Chemische Untersuchungen der Zähne von Th. Gaßmann, Zürich. Diese Zeitschrift, Bd. LV, Heft 6.

2) Chemische Untersuchungen der Zähne, II. Teil, von Th. Gaßmann, Zürich. Diese Zeitschrift, Bd. LXVIII; Heft 5.

Hoppe-Seyler's Zeitschrift f. physiol. Chemio. LXX. 
auch beim rhachitischen Knochen die Werte von Kalk, Phosphorsäure und Kohlensäure in einem bestimmten Verhältnis zueinander stehen. Untersuchen wir das Verhältnis näher, so entspricht dasselbe der Formel $\left[\mathrm{Ca}_{3}\left(\mathrm{PO}_{4}\right)_{2}\right]_{3} \mathrm{CaCO}_{3}$. Nun hat uns A. Werner ${ }^{1}$ ) über den Aufbau und die Konstitution von komplexen Verbindungen, deren Kenntnisse von außerordentlichem Interesse für den Knochenbau sind, eingehenden Aufschluß gegeben. Ich glaube an Hand meiner Analysenresultate einige Anhaltspunkte zu finden, die mit der von A. Werner geschaffenen Theorie der komplexen Verbindungen in Einklang stehen. Es betrifft dies vornehmlich solche, die derselbe Autor unter einer als Appatite zu bezeichnenden Gruppe von Verbindungen zusammenfaßte: A. Werner stellt hierfür folgende Formel auf:

$$
\left[\mathrm{Me}\left(\begin{array}{c}
\mathrm{OPO}_{3} \mathrm{Me} \\
>\mathrm{Me} \\
\mathrm{OPO}_{3} \mathrm{Me}
\end{array}\right) 3\right] \mathrm{CO}_{3}
$$

oder im Sinne einer späteren Verteilung der Säuren auf die Basen:

$$
\left.\mathrm{Ca}\left(\begin{array}{c}
\mathrm{OPO}_{3} \mathrm{Ca} \\
>\mathrm{Ca} \\
\mathrm{OPO}_{3} \mathrm{Ca}
\end{array}\right) 3\right] \mathrm{CO}_{3}
$$

Berechnen wir aus obiger Formel die Werte für Kalk, Phosphorsäure und Kohlensäure in Prozenten und damit zugleich die Verhältniszahlen auf Kalk bezogen:

$$
\begin{array}{ll}
\mathrm{Ca}=38,78 \% & \mathrm{Ca}: \mathrm{PO}_{4}: \mathrm{CO}_{3} \\
\mathrm{PO}_{4}=55,38 \% & 1: 5,8: 0,9 \\
\mathrm{CO}_{3}=5,83 \% &
\end{array}
$$

und stellen denselben die aus der Analyse gefundenen Werte gegenüber:

$$
\begin{array}{ccc}
\text { Normaler Knochen: } \mathrm{Ca}=24,48 \% & \mathrm{Ca}: \mathrm{PO}_{4}: \mathrm{CO}_{3} \\
& \mathrm{PO}_{4}=33,79 \% & 1: 5,74: 0,82 \\
& \mathrm{CO}_{3}=3,20 \% & \\
\text { Rhachitischer }{ }^{\mathrm{Ca}}=21,61 \% & \mathrm{Ca}: \mathrm{PO}_{4}: \mathrm{CO}_{3} \\
& \mathrm{PO}_{4}=30,54 \% & 1: 5,8: 0,88 \\
& \mathrm{CO}_{3}=2,90 \% &
\end{array}
$$

1) A. Werner, Berl. Ber., Bd. XL, S. 4447 (1907). 
so erkennen wir mit Deutlichkeit, daß die analytischen Zahlen den theoretischen entsprechen, bezw. das Verhältnis von Kalk zu Phosphorsäure and Kohlensäure ist das gleiche geblieben, es ist ein konstantes. Hieraus können wir den Schluß ziehen, daß dieses bleibende Verhältnis von $\mathrm{Ca}: \mathrm{PO}_{4}: \mathrm{CO}_{3}$ sowohl im gesunden, als auch im rhachitischen Knochen der Ausdruck einer komplexen Verbindung bildet, und sind wir daher außer Zweifel, daß in beiden Knochen ein und dieselbe komplexe Verbindung vorhanden sein muß. Daß diese Verbindung ringförmig geschlossenen Charakter besitzt, hat wohl seine Stütze darin, daß auch der krankhafte Knochen mit seiner Veränderlichkeit auf die Stabilität dieser Salzbildung keinen zersetzenden Einfluß auszuüben vermag. Noch erübrigt es mir, die eigentliche Verteilung der Phosphorsäure und Kohlensäure auf die Kalkbase vorzunehmen, was im Sinne der Wernerschen Formel ohne Schwierigkeit sich vollziehen läßt. Wir erhalten dementsprechend für

$\begin{array}{lcr}\text { gesunden Knochen } 60,44 \% & \\ \text { rhachitischen } & \text { } & 54,49 \%\end{array}$

der komplexen Verbindung.

$$
\left[\mathrm{Ca}\left(\begin{array}{c}
\mathrm{OPO}_{3} \mathrm{Ca} \\
\underset{\mathrm{Ca}}{\mathrm{OPO}} \mathrm{Ca}
\end{array}\right) 3\right] \mathrm{CO}_{3}
$$

Ziehen wir damit auch die Anwesenheit des Wassers in Betracht, so dürfte die entsprechende Formel etwa so lauten:

$$
\left[\mathrm{Ca}\left(\begin{array}{c}
\mathrm{OPO}_{8} \mathrm{Ca} \\
>\mathrm{Ca} \\
\mathrm{OPO}_{8} \mathrm{Ca}
\end{array}\right){ }^{2}\right] \mathrm{CO}_{8}+\mathrm{x}_{8} \mathrm{O}
$$

Sowohl beim normalen als auch beim rhachitischen Knochen verbleibt nach der Verteilung der Säuren auf die Kalkbase noch ein kleiner Rest ungesättigten Kalkes. Dieser beträgt für den normalen Knochen etwa 1\%, für den rhachitischen $0,5 \%$. Es ist dies charakteristisch deshalb, weil es uns die Möglichkeit bietet; der von A. Werner entwickelten Theorie über den Aufbau der komplexen Verbindungen die erste Anlage zu geben. Wie derselbe Autor berichtet, entstehen die oben 
angeführten komplexen Verbindungen aus den Hexolsalzen, denen die Formel ${ }^{1}$ )

zukommt.

$$
\left[\mathrm{Me}\left({ }_{\mathrm{HO}}^{\mathrm{HO}} \mathrm{Me}\right)_{3}\right] \mathrm{x}_{2}=\left[\mathrm{Ca}\left({ }_{\mathrm{HO}}^{\mathrm{HO}} \mathrm{Ca}\right)_{3}\right] \mathrm{CO}_{3}
$$

Diese hinwiederum entstehen entweder durch Anlagerung von trimolekularem Metallhydroxyd an das Neutralsalz oder durch Substitution des Wassers in den Hexahydraten der Metallsalze durch Metallhydroxydmoleküle. Der Umstand also, daß das Knochenmorgan noch kleine Mengen von Kalk zur Verfügung hat, für die kein Säureanteil mehr vorhanden ist, gibt wohl zu erkennen, daß die Möglichkeit von weiterem Knochenaufbau nicht ausgeschlossen ist. Diese Erscheinung tritt bei den Menschenzähnen noch mehr zutage, wo in einem Fall bis zu 2,5\% Kalk durch Säure nicht mehr abgesättigt werden kann. Hat nun dieser überschüssige Kalk wirklich den Zweck, als weiteres Aufbaumaterial dem Knochen dienlich zu sein, so ist es wahrscheinlich, daß noch andere Faktoren im Knochen entstehen, die uns den Werdegang eines Hexolsalzes erkennen lassen. Dies kennen wir in der Tat an einem Beispiel beobachten. Meine Analysen über die Weisheitzähne haben gezeigt, daß dieselben den größten Kalkgehalt besitzen und auch bezüglich Kohlensäure gegenüber allen andern Menschenzähnen ein Mehr von 1\% aufweisen. Mit dieser Steigerung der Kohlensäure ist zugleich eine solche des Kalkes verbunden, was darauf schließen läßt, daß sich neuerdings kohlensaurer Kalk gebildet hat. Ist dem so, so liegt die Annahme sehr nahe, $\mathrm{da} B$ sich ein Hexolsalz bilden kann. Diese Entwickelungen sollen vorerst nur zeigen, daß der Knochenaufbau in diesem Sinne recht wohl an Hand der geschaffenen Analysen denkbar ist. Inwieweit das Magnesium sich bei der Bildung eines Salzes beteiligt, läßt sich aus der Analyse nicht erkennen. Phosphorsäure und Kohlensäure kommen also nicht in Frage. Chlor ist sich im Gegensatz zum Magnesium sowohl bei den Menschenzähnen als auch beim normalen und rhachitischen Knochen in

1) A. Werner, Berl. Ber., Bd. XL, S. 442 (1907). 
seinem Gehalte so stabil geblieben, daß an eine Verbindung mit Chlor nicht zu denken ist.

Hingegen bildet das Chlor eine Verbindung mit Kalium, eventuell Natrium; diese drei Elemente weisen bis jetzt überall den gleichen Gehalt auf mit einer einzigen Ausnahme bei den Hundezähnen. Bei diesen wurde der Chlorgehalt etwas kleiner, sofort tritt die gleiche Erscheinung auch beim Kalium zutage.

Zusammenfassung der erhaltenen Ergebnisse.

Der normale Knochen enthält etwa 1\% mehr Wasser als der rhachitische. Nicht nur der Kalk, auch die Phosphorsäure und Kohlensäure vermindern beim rhachitischen Knochen ihren Gehalt. Im engen Zusammenhang steht damit die Abnahme des Wassers, was darauf schließen läßt, daß im Knochen ein neuer Fall von Hydratbildung vorliegt.

Das Verhältnis von $\mathrm{Ca}: \mathrm{PO}_{4}: \mathrm{CO}_{3}$ ist beim normalen und rhachitischen Knochen dasselbe.

Es beträgt für den normalen Knochen $1 \mathrm{Ca}: 5,74 \mathrm{PO}_{4}: 0,82 \mathrm{CO}_{3}$

$\gg>$ rhachitischen $>1 \mathrm{Ca}: 5,8 \mathrm{PO}_{4}: 0,88 \mathrm{CO}_{3}$.

Damit läßt sich unwillkürlich die Annahme verbinden, daß in beiden Knochen die gleiche komplexe Verbindung:

vorhanden ist.

$$
\left[\mathrm{Ca}\left(\begin{array}{c}
\mathrm{OPO}_{8} \mathrm{Ca} \\
\underset{\mathrm{Ca}}{\mathrm{OPO}_{3} \mathrm{Ca}}
\end{array}\right) 3\right] \mathrm{CO}_{8}
$$

Da diese komplexen Verbindungen nach $\mathrm{A}$. Werner ringförmig geschlossene Konstitution haben, infolgedessen größere Stabilität besitzen, so erklärt sich auch ihre Unveränderlichkeit im kranken Knochen.

Hieraus resultiert auch, daß die Krankheit nicht in einer Zersetzung der komplexen Salzbildung bestehen kann.

Das Magnesium erfährt im rhachitischen Knochen eine bedeutende Vermehrung. Seine Zugehörigkeit zu einem salzbildenden Körper wie Phosphorsäure, Kohlensäure und Chlorwasserstoffsäure ist nach den Analysenresultaten nicht denkbar.

Kalium, Natrium und Chlor dürften der Ernährungsflüssigkeit des Knochens angehören und sich am eigentlichen Knochenaufbau nicht beteiligen. 
Im rhachitischen Knochen ist die Menge des Glühverlustes bezw. der organischen Substanz um etwa $5 \%$ vermehrt.

Nehmen wir zu dieser Vermehrung des Glühverlustes noch diejenige des Magnesiums hinzu, so entspricht dies dem Ausfall der Kalksalzbildung im rhachitischen Knochen.

Bemerkenswert ist noch, daß in beiden Knochen der gleiche Stickstoffgehalt vorhanden ist.

Auf Grund der chemischen Untersuchung kann man sich nunmehr der Ansicht nicht verschließen, daß dem vermehrten Magnesiumgehalt in pathologischer Hinsicht eine wichtige Rolle zukommt. Er tut dies um so mehr, als ich bereits bei meiner Forschung nach den Ursachen der Zahncaries eine ähnliche Erscheinung feststellen konnte; auch da hat es sich gezeigt, daß die Zähne, die zu Caries hinneigen, bedeutend mehr Magnesium besitzen, als solche, die von dieser Krankheit nicht berührt werden. Solche übereinstimmenden Ergebnisse lassen doch deutlich erkennen, daß das Magnesium da in vermehrter Menge auftritt, wo wir Abnormalitäten in der chemischen $\mathrm{Zu}$ sammensetzung des Knochenorganes zu erwarten haben. Daß diese hemmend, störend auf den Knochenbau einwirken können, ist ersichtlich und bekräftigt unsere Annahme, daß dies Vorbedingungen für die Krankheitserscheinungen der Rhachitis und Zahncaries sein können. 